\title{
Study of the Effects of Radiation at the CERN Gamma Irradiation Facility on the CMS Drift Tubes Muon Detector for the HL-LHC on the CMS DT for the HL-LHC
}

\author{
Juan R. González Fernández* on behalf of the CMS Muon Group \\ Universidad de Oviedo, Spain \\ E-mail: jrgonzalecern.ch
}

\begin{abstract}
The CMS drift tubes (DT) muon detector, built for standing up the LHC expected integrated and instantaneous luminosities, will be used also in the High Luminosity LHC (HL-LHC) at a 5 times larger instantaneous luminosity and, consequently, much higher levels of radiation, reaching about 10 times the LHC integrated luminosity. Initial irradiation tests of a spare DT chamber at the CERN gamma irradiation facility (GIF++), at large ( $\mathrm{O}(100)$ ) acceleration factor, showed aging effects resulting in a degradation of the DT cell performance; however, full CMS simulations have shown almost no impact in the muon reconstruction efficiency over the full barrel acceptance and for the full integrated luminosity. A second spare DT chamber was moved inside the GIF++ bunker in October 2017. The chamber was being irradiated at lower acceleration factors, and only 2 out of the 12 layers of the chamber are switched at working voltage when the radioactive source is active, being the other layers in standby. In this way the other nonaged layers are used as reference and as a precise and unbiased telescope of muon tracks for the efficiency computation of the aged layers of the chamber, when set at working voltage for measurements. An integrated dose equivalent to two times the expected integrated luminosity of the HL-LHC run has been absorbed by this second spare DT chamber and the final impact on the muon reconstruction efficiency is under study. Direct inspection of some extracted aged anode wires presented a melted resistive deposition of materials. Investigation on the outgassing of cell materials and of the gas components used at the GIF++ are underway. Strategies to mitigate the aging effects are also being developed. From the long irradiation measurements of the second spare DT chamber, the effects of radiation in the performance of the DTs expected during the HL-LHC run will be presented.
\end{abstract}

European Physical Society Conference on High Energy Physics - EPS-HEP2019 -

10-17 July, 2019

Ghent, Belgium

${ }^{*}$ Speaker. 


\section{Introduction}

During the LHC Run 2 the CMS experiment has recorded an integrated luminosity of more than $165 \mathrm{fb}^{-1}$. This enormous amount of data has allowed the CMS collaboration to explore a wide range of particle physics phenomena including the discovery of the Higgs boson in 2012, however no strong sign of new physics has been observed. An upgrade of the LHC is planned to extend the sensitivity of BSM searches, the HL-LHC.

The HL-LHC will provide pp collisions at an instantaneous luminosity about a factor 5 larger than the current LHC luminosity. It has a design value of $5 \times 10^{34} \mathrm{~cm}^{-2} \mathrm{~s}^{-1}$ and an ultimate value, expected to be reached in the last years of the phase II, of about $7.5 \times 10^{34} \mathrm{~cm}^{-2} \mathrm{~s}^{-1}$. The HL-LHC is planned to run for a decade, starting on 2026 , collecting about $3000 \mathrm{fb}^{-1}$ of pp collisions.

The continuous exposure to radiation of the DT subsystem for more than a decade will affect the performance of the detector, that will have to deal with an ageing effect produced by the damage to the detector and electronics produced by the radiation. The effects of the ageing on the performance of the DT have been preliminary estimated [1]. In order to characterize the expected evolution of the performance along the HL-LHC run, a MB2 chamber was introduced into the Gamma Irradiation Facility (GIF++) bunker, located at CERN, where it was irradiated using an intense ${ }^{137} \mathrm{Cs}$ source. The MB2 chamber is a DT station consisting of 12 layers, grouped into 3 superlayers (SL), with about 60 DT cells per layer.

\section{Irradiation and dose measurements}

The MB2 chamber at GIF++ is irradiated at an acceleration factor of 10 with respect to the expected radiation rate during the HL-LHC. During this irradiation only 2 out of 12 layers (layers 1 and 4 of SL 1) are kept at a high voltage of $3550 \mathrm{~V}$, while the rest of the chamber is kept in standby, so no ageing effect is produced, so the performance of non-aged layers is taken as reference.

The amount of radiation received by the chamber is quantified with dosimetric measurements. At CMS, the integrated dose received by any of the DT stations is proportional to the integrated luminosity along the time. A conversion factor between these two quantities is derived to extrapolate from the integrated dose at GIF++ to the expected integrated luminosity at CMS, using the most irradiated chambers, MB1 in the external wheels, as reference.

The MB2 was irradiated during two periods, each of them of about 13 weeks. The evolution of the absorbed dose by the chamber is shown in figure 1. After the full irradiation period, the chamber received an amount of radiation equivalent to twice the expected dose at the end of the HL-LHC.

\section{Measurement of the hit efficiency}

The hit efficiency is measured using cosmic muons, triggered using SL2 and SL3, or a muon beam, using external scintillators to trigger the events. The efficiency is calculated for a given layer in SL1 by selecting segments with associated hits in 4 layers of SL3 and counting the number of times that a hit is found in the expected position in that test layer. A schematic explanation of this calculation is shown in figure 2 . 


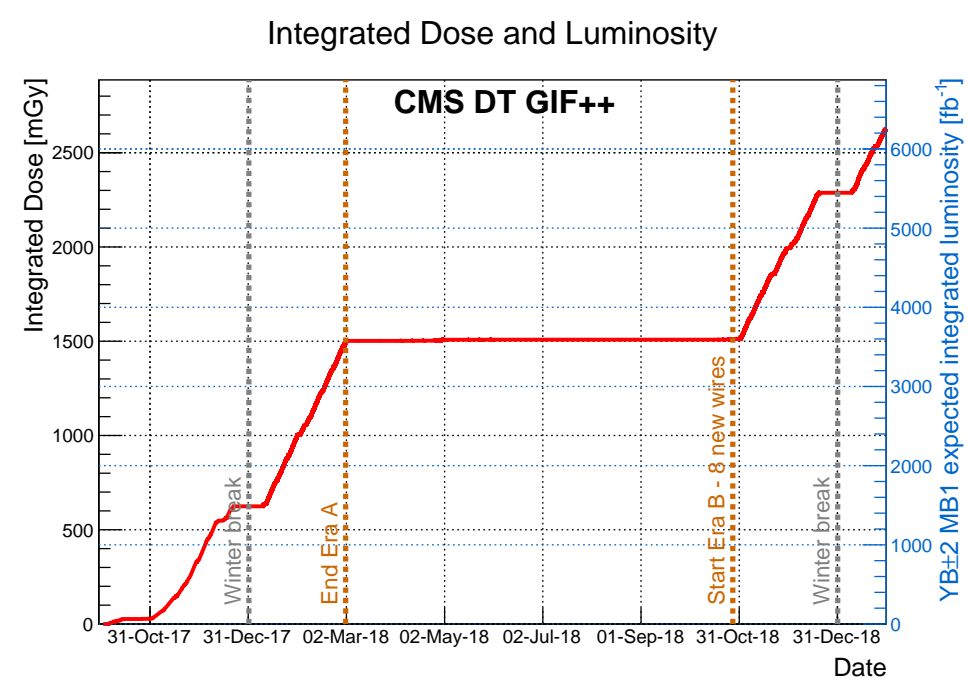

Figure 1: Integrated dose (mGy) as a function of time for the full irradiation period of the CMS MB2 spare chamber at GIF++. The vertical grey lines correspond to the 2017 and 2018 winter breaks when the chamber is kept off and the vertical orange lines mark the two irradiation and data-taking eras. The axis on the right shows the equivalent expected luminosity for MB1 chambers in the external wheels for the HL-LHC.

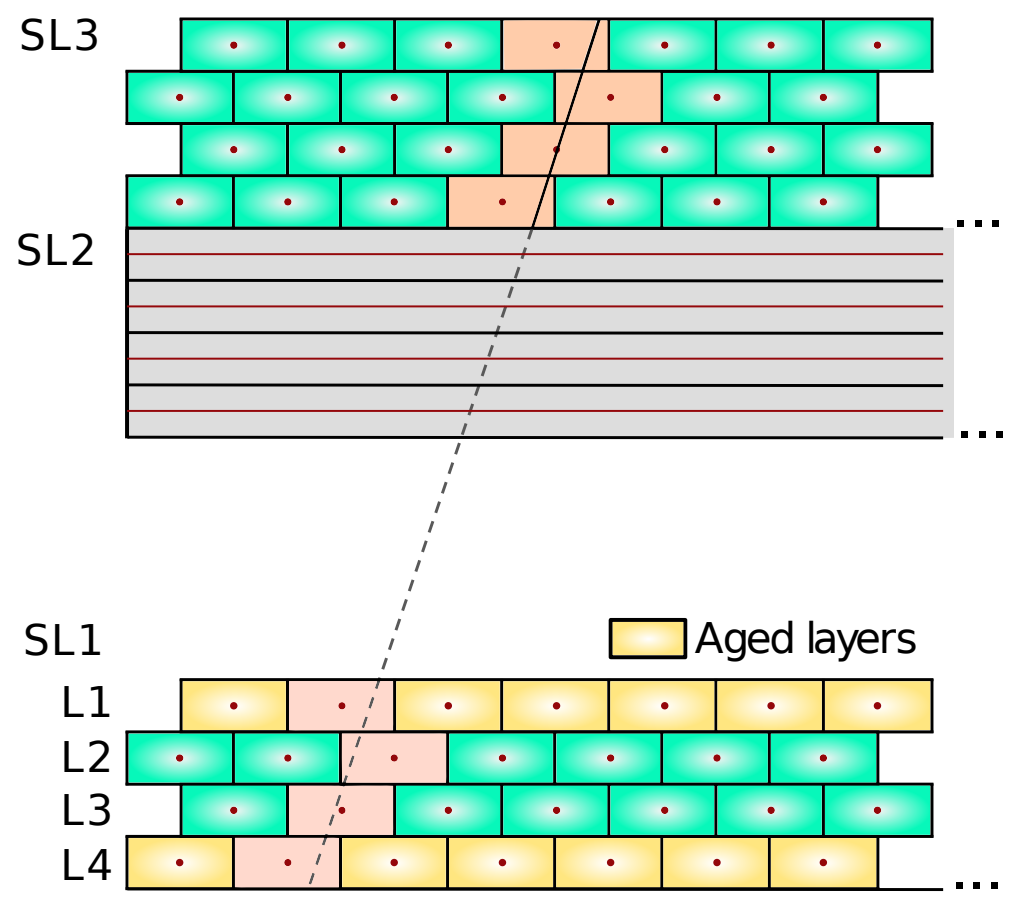

Figure 2: Layout of the efficiency calculation. SL3 is used to reconstruct segments and calculate the expected hit position in SL1. 


\section{Evolution of the hit efficiency}

The efficiency was measured along the irradiation period using cosmic muons with no background rate (source off). The evolution of the efficiency up to an expected integrated luminosity of about $6000 \mathrm{fb}^{-1}$ is shown in figure 3. A small decrease of the efficiency is observed at zero source rate, even after integrated twice the expected radiation at the end of the HL-LHC.

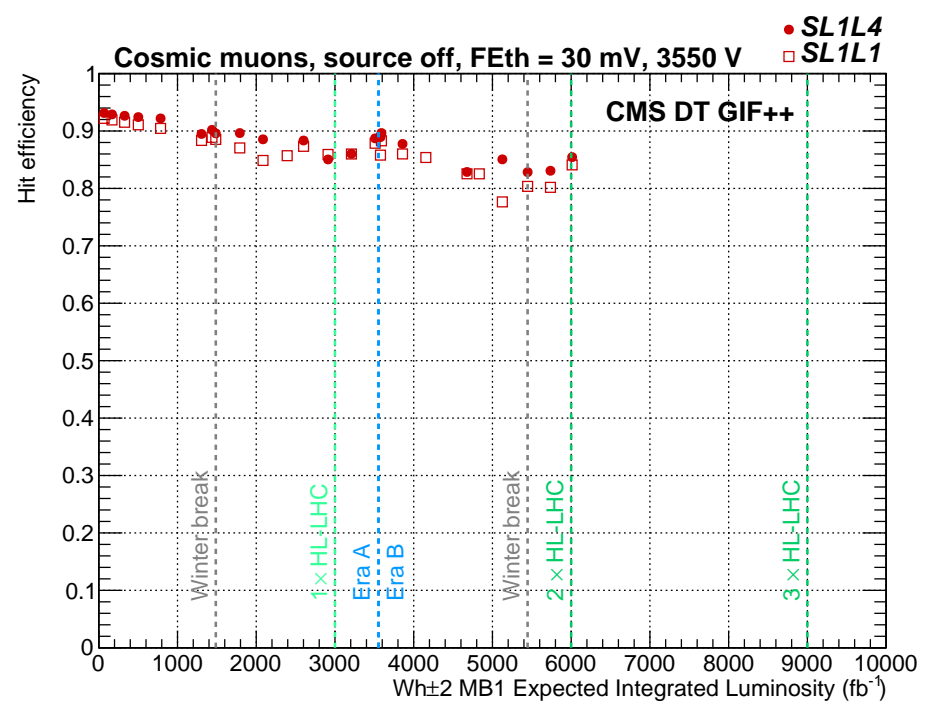

Figure 3: Evolution of the efficiency as a function of the expected integrated luminosity for cosmic muons and source off.

The evolution of the efficiency is also measured as a function of the expected integrated luminosity using cosmic muons for a background rate similar to the one expected at the HL-LHC, shown in figure 4 (left). In this case we observe a large decrease of the efficiency for the aged layers. Moreover, the efficiency is measured as a function of the expected instantaneous luminosity using muons from a beam after the chamber absorbed an integrated luminosity of $3660 \mathrm{fb}^{-1}$, shown in figure 4 (right). The two distributions are fitted using a exponential model.

Using the fitted curves, a hit efficiency of 0.62 is expected for the most irradiated chambers after the HL-LHC, applying a safety factor 2 on both expected integrated and instantaneous luminosity. This result is scaled to the full CMS DT system, using data of absorbed charge during 2018 and extrapolating the conditions to the ones expected at the HL-LHC in order to obtain an extrapolation factor for each DT station. The expected efficiency loss for the rest of chambers is much smaller than for the MB1 chambers on the external wheels, with expected efficiencies up to 0.80 for other MB1 and MB4 chambers and efficiencies larger than 0.9 for MB2 and MB3 chambers.

\section{Muon reconstruction efficiencies}

To test the effect of the efficiency loss on the CMS muon reconstruction, a simulation has been performed in which the efficiencies of each DT station were reduced to the expected value 

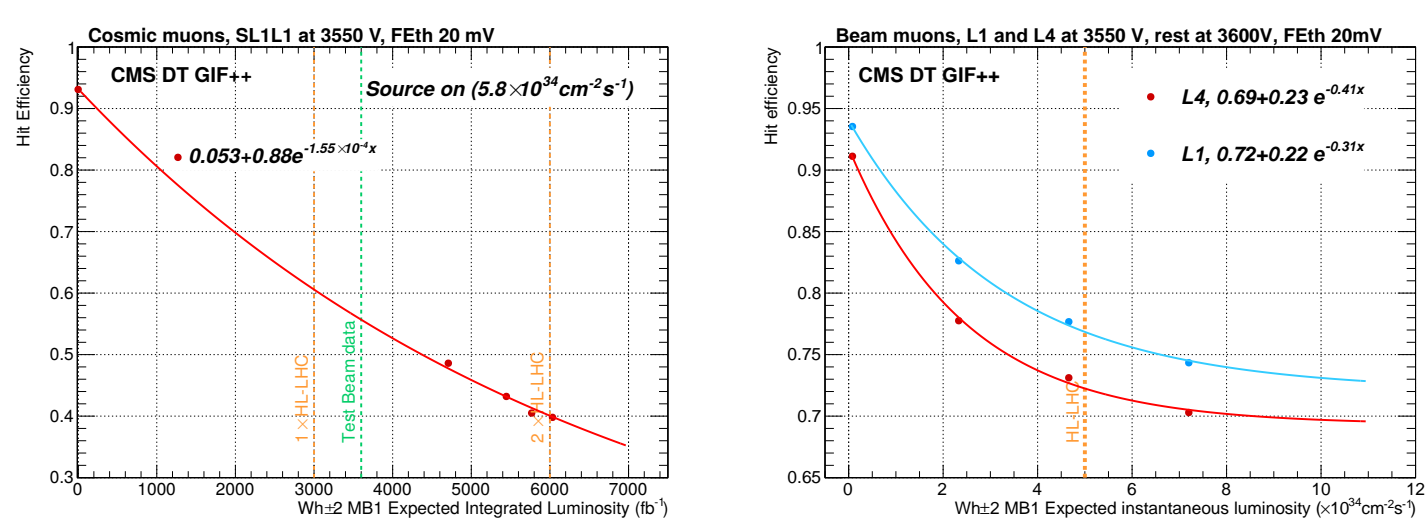

Figure 4: Evolution of the efficiency as a function of the expected integrate luminosity using cosmic muons (left) and as a function of the instantaneous luminosity using muons from a muon beam (right). The distributions are fitted using exponential models.

at the end of the HL-LHC considering a safety factor 2 on expected integrated and instantaneous luminosity. To observe the effect of the ageing, the efficiencis of reconstructing standalone muons with and without ageing effects is compared as a function of the pseudorapidity in figure 5 .

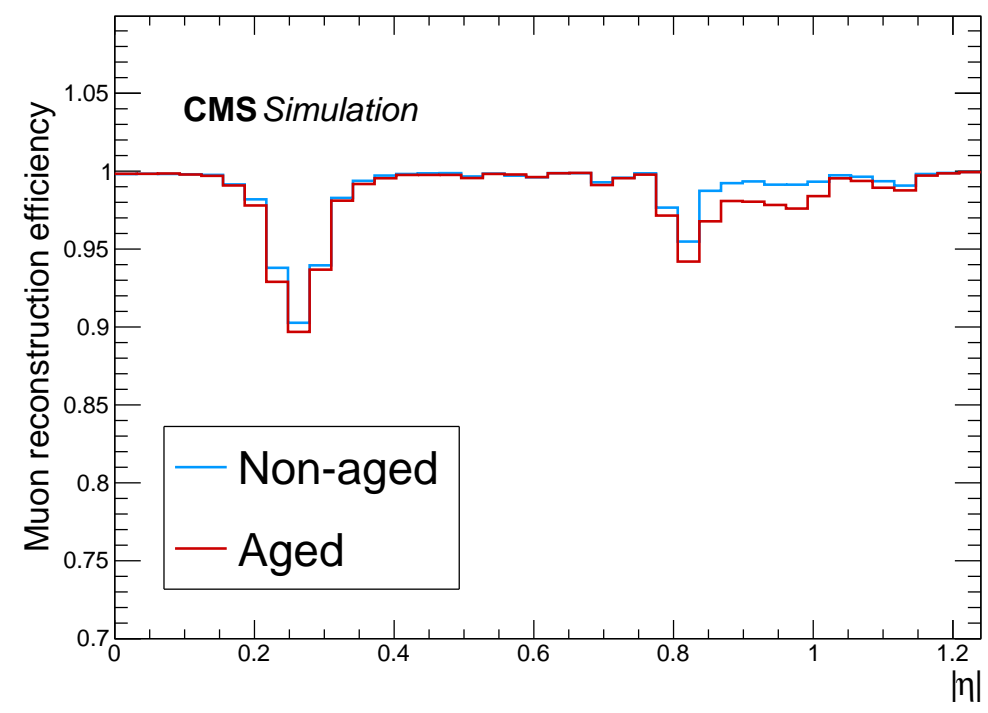

Figure 5: Expected reconstruction efficiency from simulation as a function of the pseudorapidity for standalone muons, for aged and non-aged scenarios.

The observed effect of the ageing on the CMS standalone reconstruction is small due to the redundancy of the system. A maximum efficiency loss of about $2 \%$ is observed at $0.8<|\eta|<1$.

\section{References}

[1] CMS Collaboration, The Phase-2 Upgrade of the CMS Muon Detectors, CMS-TDR-016. 